\title{
L'ARGOTIER EST-IL UN INVERTÉBRÉ ? Y A PEU D'OS DANS L'ARGOT
}

\author{
Jean-François SABLAYROLLES ${ }^{1}$ \\ USPC et HTL UMR 7597, Paris
}

\begin{abstract}
En): Names of bones seem to be used rarely in French slang, contrary to other parts of the body. Four French sources (two slang dictionaries and two academic studies of slang) were examined and the words denoting body parts extracted, confirming that names of bones are indeed hardly ever mentioned. It turns out that the inner body parts in general, and not just bones, are rarely used for slang, unless they have some visible external manifestation. Those bone names that are found in slang are sometimes scientific words, presumably used for expressive purposes.
\end{abstract}

Keywords (En): semantics; theta roles; metalexicography; body-part name; slang

Mots-clés (Fr) : Sémantique ; rôles sémantiques ; métalexicographie ; nom de partie du corps ; argot

DOI : 10.32725/eer.2021.001

\section{Introduction}

Le titre de la contribution est un peu provocateur, et peut-être aussi risqué s'il parvenait aux oreilles des gens du milieu qui pourraient prendre comme une insulte d'être traités d'invertébrés. Il a été inspiré par cette constatation de la quasi-absence de noms d'os dans les sources secondaires d'argot consultées. Si des dénominations argotiques ou familières de nombre de parties du corps me venaient spontanément en tête, je n'arrivais pas à trouver des noms d'os. J'ai voulu vérifier si cette incapacité était due à mon ignorance ou reflétait une absence réelle. La vérification a été conduite sur quatre sources secondaires, facilement consultables et pour la plupart reconnues : le livre de P. Guiraud, L'argot dans la $6^{\text {ème }}$ éd. de 1973, une réédition de 2006 du dictionnaire de Colin et alii de 1990, La $1{ }^{\text {ère }}$ éd. de Comment tu tchatches de J.-P. Goudaillier (1997), et un dictionnaire d'argot en ligne, celui de Mondouiis, à la fiabilité moindre que les autres sources. Mes intuitions sont sorties confortées de cette étude comparative. Si les parties du corps sont bien représentées dans ces sources, les os ne le sont guère (2), alors qu'ils sont en fait très nombreux (1). Des hypothèses seront avancées sur cette quasi-absence et les caractéristiques des rares os qui font exception seront abordées (3). La présentation de quelques noms savants d'os en relation avec l'argot ou dans des dictionnaires (4) précédera la conclusion.

\footnotetext{
${ }^{1}$ Jean-François Sablayrolles (25.04.1951 - 11.12.2020) n'a malheureusement pas eu le temps de relire les épreuves de son article. C'est son collègue et ami, John Humbley, qui a gentiment accepté d'effectuer les dernières corrections et de compléter certaines informations telles que le résumé (en anglais) et les mots-clés pour les deux langues, l'anglais et le français.
} 


\section{Les nombreux os du corps humain}

Afin de mettre en valeur la très faible proportion de noms d'os en argot, un bref rappel sur les os humains s'impose pour ceux qui, ayant oublié leurs cours de SVT (les leçons de choses d'antan), n'auraient plus d'idées précises sur un ordre de grandeur de leur nombre, qui est assez élevé. Les informations qui suivent ont été prises dans des encyclopédies en ligne début 2020.

On compte 206 os constants distincts chez un humain adulte, alors qu'un enfant a 350 os mous dont certains se soudent lors de la croissance. Ils forment deux ensembles: le squelette axial qui constitue la charpente du corps humain et le squelette appendiculaire qui comprend les bras et les jambes. Les os humains se distinguent en trois types: les os courts, comme les vertèbres, les carpiens du poignet..., les os longs comme le fémur, l'humérus, le tibia, le radius... et les os plats comme l'omoplate, l'os iliaque, l'os de la voûte du crâne...

Les os se répartissent ainsi : 26 os de la colonne vertébrale, 8 os au niveau du crâne, 14 os au niveau de la face, 25 au niveau du sternum et des côtes, 64 dans les membres supérieurs, 62 dans les membres inférieurs. Remarquons que l'addition de tous ces nombres donne 199 pour 206 os annoncés. Il en manquerait 7 ! Mais il existe des os surnuméraires, inconstants. Le squelette axial comprend, dans Wikipédia, 88 os et le squelette appendiculaire 136, ce qui fait un total de $224^{2}$. Mais ces légers flottements numériques n'ont pas d'incidence sur les ordres de grandeur que nous allons examiner au niveau du lexique pour les dénominations des os parmi les parties du corps et les nomenclatures complètes des quatre sources retenues. Tous les pourcentages ont été calculés sur la base de 224 os.

\section{Nombre et pourcentage des mots du corps et des os dans quatre sources secondaires}

\subsection{Guiraud [1956] 1973}

Dans le Que sais-je ? de P. Guiraud, les différentes parties du corps abondent. Ce qui s'explique par le fait que «c'est surtout le corps qui est la grande source des images » (p. 43, dans la partie consacrée à la concrétisation de l'abstrait).

\footnotetext{
${ }^{2}$ Voici un extrait de Wikipédia (23/06/2021) : « À l'âge adulte, il comprend 206 os constants. Ces os constants peuvent être divisés en 204 os squelettiques et 2 os sésamoïdes - soit 115 os différents (respectivement, 114 et 1). Avec les os surnuméraires, inconstants, on peut dénombrer jusqu'à 224 os constants, que l'on peut diviser en 212 os squelettiques et 12 os sésamoïdes - soit 128 os différents (respectivement, 122 et 6). » La consultation des articles «os », «squelette » et « vertébré » de l'Encyclopcedia Universalis (1989) apporte de nombreuses informations scientifiques très érudites, mais aucun éclairage sur le nombre des os humains et leur classement en plats, courts et longs. On y apprend en revanche qu'ils se différencient en tissus osseux primaires : os acellulaire et os cellulaire, et en os secondaires remaniés : os haversien, os spongieux remanié et os spongieux rendu compact. Ces informations sont fort éloignées de la connaissance commune que l'on peut avoir sur les os et ne sont pas d'un grand intérêt pour la présente étude, sauf la distinction entre le squelette axial (formé du crâne et du squelette postcéphalique ainsi que du squelette axial postcéphalique) et le squelette zonaire et appendiculaire (formé des ceintures pectorales et pelviennes ainsi que des membres).
} 
L'auteur indique que la liste des images du dictionnaire de Lacassagne permet de se faire une idée du pittoresque de l'argot moderne (p. 85). Apparaissent en effet 27 parties du corps, mais au sein de celles-ci, les os sont très peu présents. Ils sont passés en gras dans la liste suivante, dans laquelle les dénominations argotiques sont énumérées sauf quand elles sont très nombreuses, auquel cas c'est ce nombre qui est indiqué. Les pages où sont cités ces mots sont indiquées entre parenthèses.

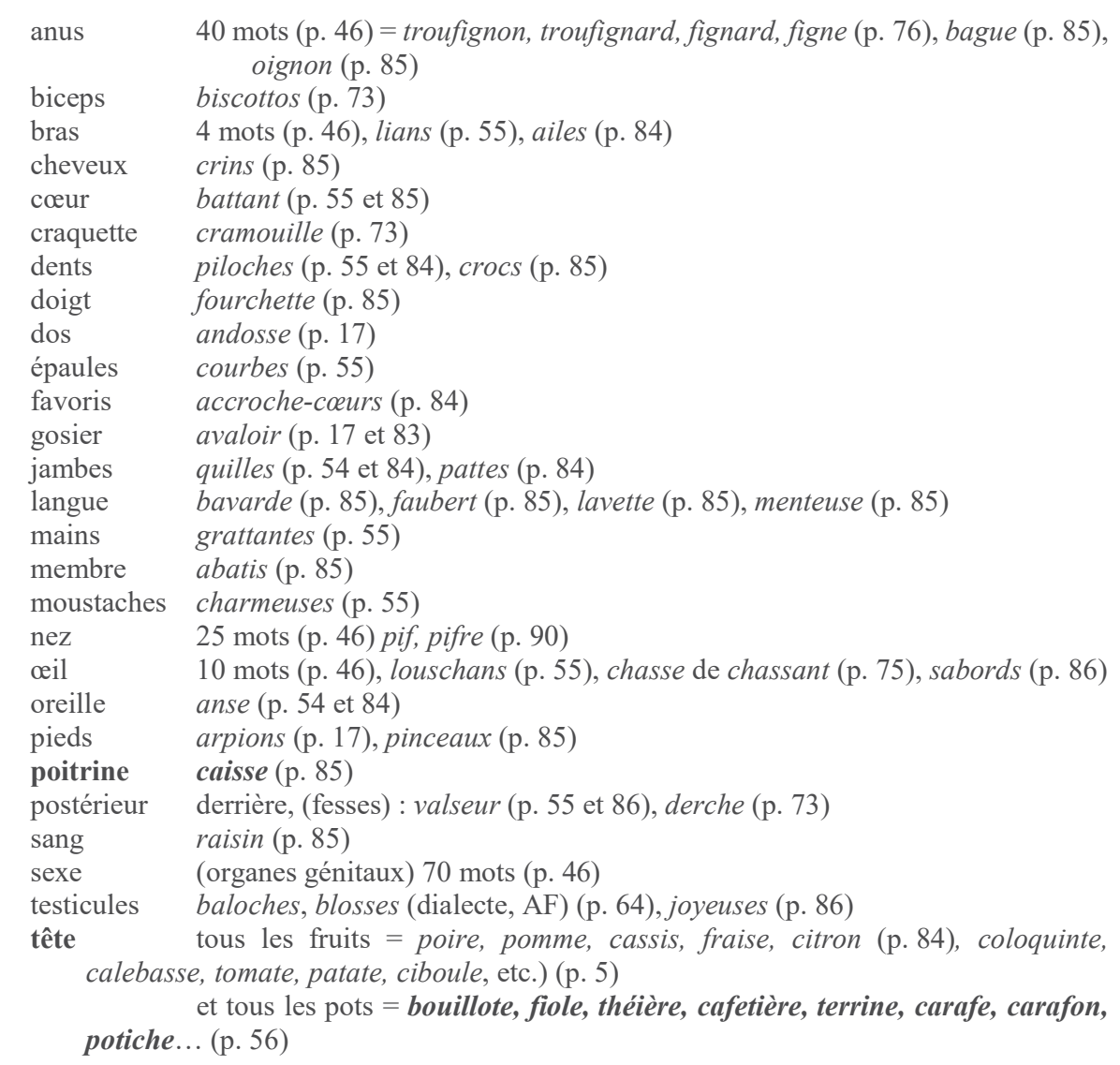

On ne relève donc que très peu de noms d'os (et aussi très peu de noms de muscles) dans l'index des mots argotiques cités. Mais cet index est incomplet et l'auteur fait état de tabous sur certains mots évoqués mais non cités : «omis[sion] des plus obscènes » (p. 86). On ne voit cependant pas quelles raisons de bienséance justifieraient des omissions dans les dénominations des os. Ils sont deux dans l'inventaire.

(i) Même si la poitrine n'est pas un os à proprement parler, sa dénomination argotique caisse incite à la considérer comme tel. Il s'agit en effet, d'une manière métaphorique, d'un contenant rigide évoquant la cage thoracique constituée des côtes. Le mot cage relève d'ailleurs d'une métaphore, complètement lexicalisée, 
semblable à celle à l'œuvre dans caisse : un contenant rigide ${ }^{3}$. Plusieurs acceptions, fondées en partie sur des métonymies coexistent pour poitrine. Par proximité avec l'aspect extérieur, présent dans tour de poitrine pour les vêtements, poitrine peut dénommer les seins, comme dans poitrine opulente. En revanche par contiguïté avec l'intérieur, le contenu, poitrine dénomme surtout les poumons (et parfois aussi le cœur). Si l'expression $s$ 'en aller de la poitrine (ainsi que le nom suffixé poitrinaire) est vieillie, l'expression argotique synonyme $s$ 'en aller de la caisse pour mourir de problèmes pulmonaires, de tuberculose, est toujours utilisée.

(ii) On relève une série synonymique de tête pour tous les mots dénommant un contenant rigide, solide, qui peut se briser, avec toutes sortes de pots. Cette liste de 8 noms, qui pourrait sans aucun doute être élargie, correspond bien aux séries synonymiques décrites par M. Schwob et G. Guieysse (1889: $33 \mathrm{sq}$ ) citées par P. Guiraud (p. 56) et aussi par A. Meillet ([1906: 247] $2015: 325)$.

En tout état de cause, c'est très peu : 9 dénominations pour 2 os (sur les 224 existants, ils représentent $0,89 \%$ ) et c'est très peu aussi par rapport à l'index des mots argotiques et populaires de la fin de l'ouvrage qui compte, sauf erreur, 1045 items. Les noms d'os représentent $0,86 \%$ de l'ensemble des mots populaires ou argotiques traités dans le livre. La proportion est infime.

Le nombre de noms de parties du corps s'élève à au moins 69 , sans compter d'éventuels oublis (le sens n'est pas toujours donné et les listes sont incomplètes, comme l'auteur le reconnaît lui-même, pour des raisons de place et aussi par exclusion volontaire de certains mots qui pourraient choquer, voir supra). Ces mots représentent 6,6\% de l'ensemble des mots de l'index et les 9 dénominations d'os sur les 69 mots du corps représentent $13 \%$. Étant donné l'importance du squelette dans le corps, et sa longévité éventuelle après la mort après putréfaction des chairs, ce pourcentage n'est pas très élevé.

\subsection{Dictionnaire de Colin et alii [1990] 2006}

D'après les informations fournies par les auteurs mêmes, la nomenclature compte 10000 entrées et 2000 sous-entrées. La consultation du dictionnaire inverse français-argot placé à la fin du livre (p. $855 \mathrm{sq})$ donne les résultats suivants pour l'étude des noms des os en argot, par rapport à l'ensemble et par rapport aux mots du corps ${ }^{4}$ : ont été relevés 1055 mots d'argot (ou du français populaire) de mots du corps pour 64 parties du corps. Ils représentent $8,8 \%$ de l'ensemble de la nomenclature (entrées et sous-entrées). Comme dans le relevé précédent, ces mots sont énumérés sauf quand leur nombre est trop grand, auquel cas seul ce nombre est indiqué. Il permet de voir quelles parties du corps sont privilégiées par les argotiers. Les noms d'os sont passés en gras.

\footnotetext{
${ }^{3}$ Notons néanmoins que tous les mots de contenant rigide employés par métaphore pour des parties du corps en argot ne dénomment pas des os, comme le montrent bidon, buffet, placard, etc. pour le ventre. Dans ce cas, c'est uniquement le trait 'contenant' qui est retenu à l'exclusion de celui de 'rigidité'.

${ }^{4} \mathrm{La}$ fermeture des bibliothèques parisiennes n'a pas permis l'achèvement de la consultation de la nomenclature principale. Mais le dépouillement jusqu'à la lettre -q- ne modifie pas sensiblement les conclusions que permet de tirer l'exploitation de l'index inverse.
} 




${ }^{5}$ Boîte au sel n'apparaît pas dans l'index alors que c'est une entrée de la nomenclature principale comme synonyme de tête, cerveau, crâne. 


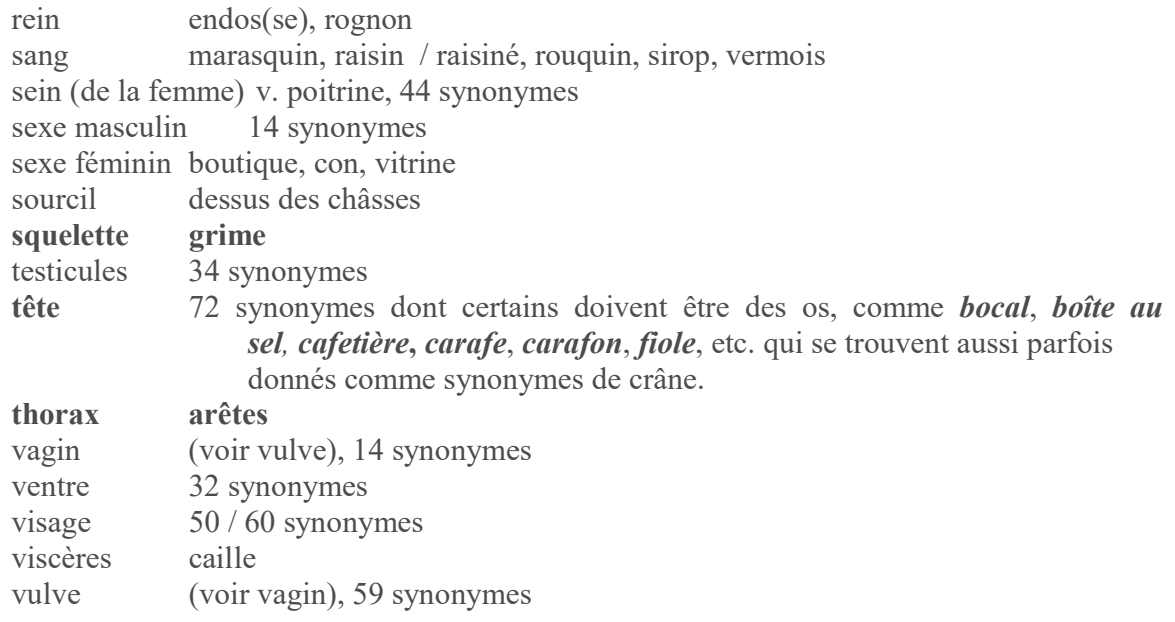

Parmi les parties du corps, si on inclut la tête qui double en partie le crâne et que l'on ne compte qu'une fois cafetière présent pour ces deux entrées, 7 os sont représentés par 20 mots argotiques :

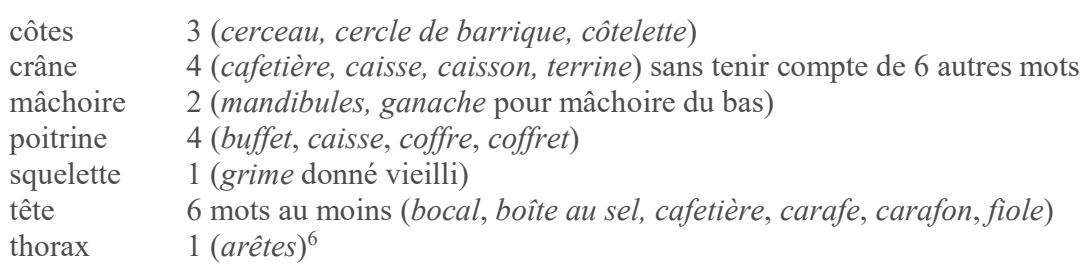

Ces 20 dénominations d'os représentent 1,9\% de l'ensemble des dénominations des parties du corps du dictionnaire, proportion infime, à peine supérieure à celle du corpus précédent. Les 7 os représentent $11 \%$ des 64 parties du corps humain qui ont au moins une dénomination populaire ou argotique. Ils représentent 3,1\% des 224 os d'un corps humain adulte. Si l'on se fie à ce vaste recueil de mots populaires et argotiques, les argotiers ne semblent pas se préoccuper beaucoup de leur squelette, ou de celui des autres. Mais il est remarquable de noter que les mots os et osselets sont eux-mêmes des mots d'argot. Employé seul os signifie «anus » (l'avoir dans l'os), et avec l'expansion à moelle, il signifie «nez» ou « pénis ». Quant à osselets, ce mot dénomme les doigts ou les dents.

\subsection{Goudaillier 1997}

Dans son dictionnaire consacré au français contemporain des cités, J.-P. Goudaillier indique 31 dénominations de parties du corps pour 7 parties du corps distinctes, dont 29 pour 5 parties liées à la sexualité, sur environ 700 entrées extraites

\footnotetext{
${ }^{6}$ En fait les arêtes ne pouvant guère être autre chose que les côtes, ce mot aurait pu être intégré dans le premier groupe, mais ce sont bien les mots thorax et dos qui apparaissent comme équivalents dans le dictionnaire.
} 
d'une base de données de 1300 items à l'époque (1997, date de la première édition). Les mots de parties du corps représentent $4,4 \%$ de la nomenclature.

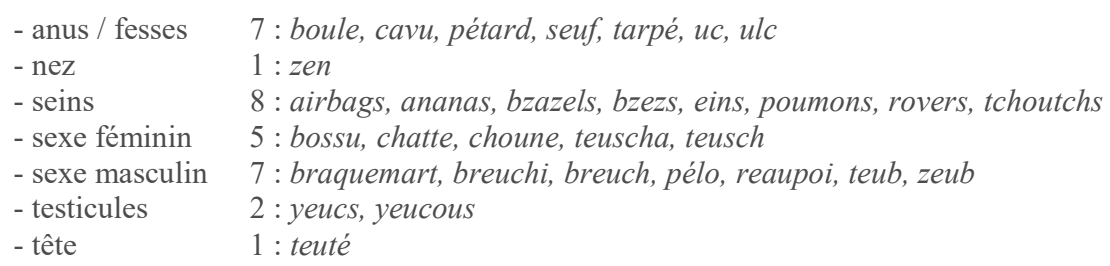

Mais on ne relève aucun nom d'os dans cet ensemble, si ce n'est la tête avec le verlan teuté qui n'indique pas directement qu'il s'agit d'un os, comme les métaphores des récipients solides (cafetière, carafon, etc.). Si les argotiers traditionnels ne se préoccupent que peu des os, la situation est encore pire pour les jeunes de cité qui semblent ne pas s'en préoccuper du tout. Leur centre d'intérêt pour le corps semble se focaliser, mais ce n'est pas nouveau, sur les parties sexuelles. Seules deux dénominations pour le nez et la tête ne font pas partie de cet ensemble. Les deux sont du verlan comme d'ailleurs beaucoup des dénominations relevées dans ce dictionnaire, qui témoigne d'un renouvellement dans les manières de s'exprimer de certains groupes sociaux.

\subsection{Dictionnaire d'argot en ligne de Mondouïs}

Pour élargir le champ des données à comparer et comme internet offre de nombreuses ressources, une a été choisie un peu au hasard afin de l'exploiter. Comme pour les corpus précédents, ont été relevés systématiquement tous les mots du corps et passés en gras les noms d'os.

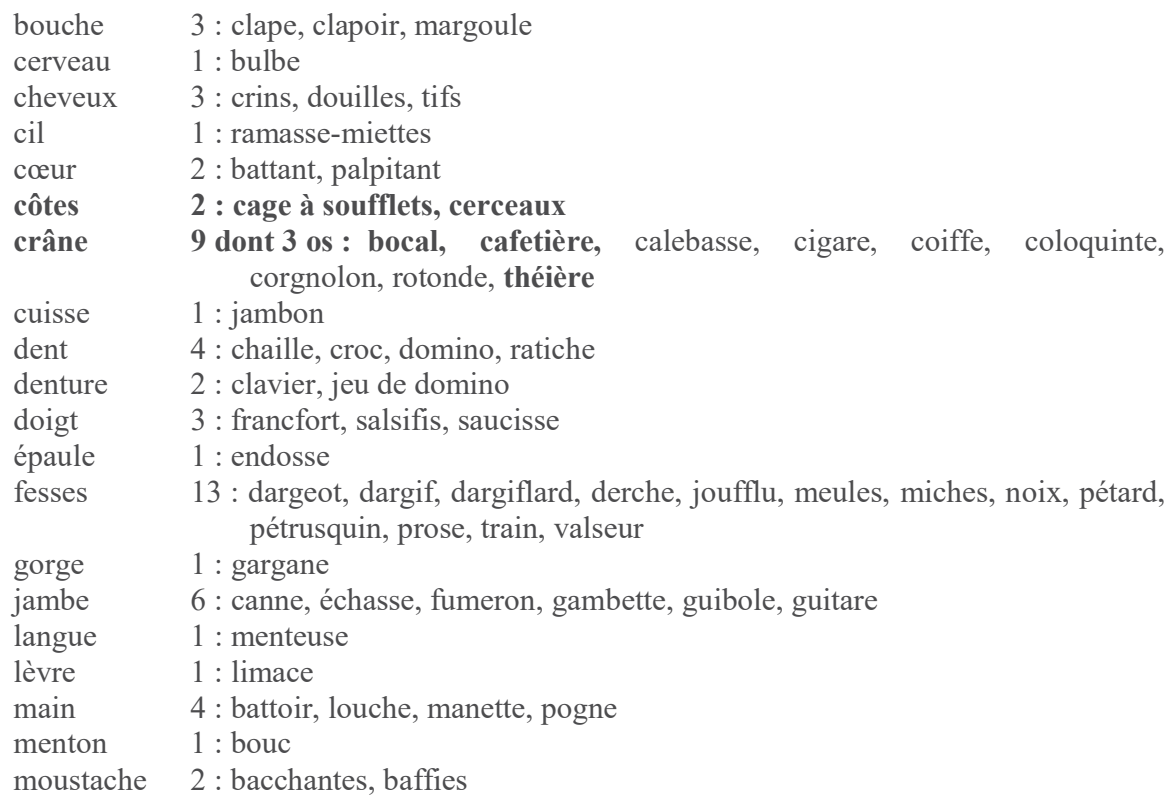




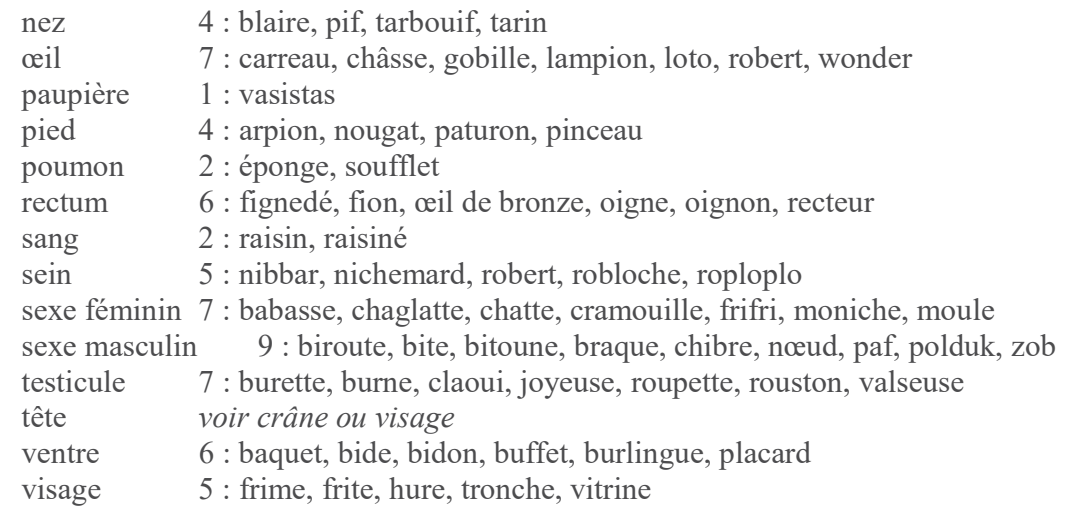

L'auteur annonce 849 mots (argotiques ou populaires) pour 345 entrées de mots du français courant. Ils ont été découverts, nous dit l'auteur, dans la cour de récré (Paris 20 ${ }^{\mathrm{ème}}$ ) dans les années 50, dans des lectures (Auguste Lebreton, San Antonio...) et forment un « joyeux mélange ».

On relève dans cet inventaire 35 mots du corps du français conventionnel, ce qui représente $10,15 \%$ des entrées. On compte 124 mots argotiques ou populaires de noms de parties du corps, ce qui représente $14,6 \%$ des mots populaires ou argotiques du dictionnaire. Dans cet ensemble on ne relève que 2 os, les côtes et le crâne, avec 11 mots argotiques et populaires, mais seulement 5 noms d'os. Il y en a 2 pour les côtes et 3 pour le crâne, les autres sont des métaphores d'un autre type : forme sphérique ou localisation supérieure pour la tête, par exemple. Ces 2 os représentent 5,7\% des 35 mots du corps et seulement $0,58 \%$ des entrées des mots courants de cet inventaire, ce qui est très peu. Les 5 noms argotiques d'os représentent $4 \%$ des mots argotiques des parties du corps et seulement $0,59 \%$ de tous les mots d'argot du dictionnaire, ce qui est encore une proportion très faible. Et les deux os représentent $0,89 \%$ des 224 existants dans le corps humain adulte, chiffre identique à ceux trouvés dans les corpus précédents, à l'exception de celui de Goudaillier où les os étaient complètement absents.

\section{Hypothèses sur la rareté des noms d'os, par ailleurs nombreux}

\subsection{Une quasi-absence avérée des noms d'os argotiques ou populaires}

Un tableau synoptique reprenant plusieurs données chiffrées et pourcentages précède quelques commentaires à leur sujet.

\begin{tabular}{|l|c|c|c|c|}
\hline & Guiraud & Colin & Goudaillier & Mondouïs \\
\hline nb noms & $69 / 1045$ & $1055 / 12000$ & $31 / 700$ & $124 / 849$ \\
parties du & $6,6 \%$ & $8,8 \%$ & $4,4 \%$ & $14,6 \%$ \\
$\begin{array}{l}\text { corps / } \\
\text { nomenclature }\end{array}$ & & & & \\
\hline $\begin{array}{l}\text { nb noms } \\
\text { parties du } \\
\text { corps / nb }\end{array}$ & $69 / 27$ & & $31 / 7$ & $124 / 35$ \\
\hline
\end{tabular}




\begin{tabular}{|l|c|c|c|c|}
\hline $\begin{array}{l}\text { parties du } \\
\text { corps }\end{array}$ & & $?^{7}$ & & \\
\hline $\begin{array}{l}\text { nb os / nb } \\
\text { parties du } \\
\text { corps }\end{array}$ & $2 / 27$ & $7 / 64$ & $0 / 7$ & $2 / 35$ \\
\hline $\begin{array}{l}\text { nb des noms } \\
\text { d'os / nb noms }\end{array}$ & $9 / 69$ & $11 \%$ & $0 \%$ & $5,7 \%$ \\
parties du & $13 \%$ & $1,9 \%$ & $0 / 31$ & $5 / 124$ \\
corps & & & & $4 \%$ \\
\hline nb noms d'os / & $9 / 1045$ & $20 / 12000$ & $0 / 700$ & $5 / 849$ \\
nomenclature & $0,86 \%$ & $0,17 \%$ & $0 \%$ & $0,59 \%$ \\
\hline $\begin{array}{l}\text { nb os / 224 os } \\
\text { humains }\end{array}$ & $2 / 224$ & $7 / 224$ & $0 / 224$ & $2 / 224$ \\
& $0,89 \%$ & $3,12 \%$ & $0 \%$ & $0,89 \%$ \\
\hline
\end{tabular}

Le nombre d'os présent dans ces quatre sources va de 0 à 7 en passant par 2 (dans 2 sources) pour 224 os du corps humain. Les pourcentages s'échelonnent entre $0 \%$ et 3,12\%. La différence de volume n'est pas seule en cause dans ces différences numériques; joue également la différence de projets : des dictionnaires généraux d'argot, aussi exhaustif et scientifique que possible pour Colin et alii, plus restreint et subjectif pour Mondouïs, face à un dictionnaire spécialisé dans le parler des jeunes de banlieue contemporains (Goudaillier) et une étude sur l'argot, qui ne se présente comme un dictionnaire, mais dont l'index joue un rôle approchant (Guiraud). La comparaison de ces quatre sources montre, de manière unanime, que la très grande majorité, pour ne pas dire la quasi-totalité des os sont ignorés des argotiers. Leur présence constitue l'exception.

Par rapport à la nomenclature de chaque source, les noms d'os représentent de $0 \%$ à $0,86 \%$ de l'ensemble des mots répertoriés. Les pourcentages sont proches dans toutes les sources et extrêmement faibles. Cette faiblesse est la conséquence de la constatation précédente du nombre restreint d'os dans l'argot et aussi du fait de la rareté de dénominations synonymiques pour ceux-ci, ce qui contraste avec l'inflation de termes argotiques pour certaines parties du corps ou activités, comme celles qui relèvent du domaine de la sexualité par exemple. Même la série, ouverte il est vrai, des noms de pots ou récipients pour dénommer la boîte crânienne ne présente, dans l'usage courant, qu'un nombre restreint d'unités.

Par rapport aux nombres des parties du corps dans chacune des sources secondaires, les nombres d'os vont de $0 \%$ à $11 \%$, ce qui ne représente pas, là non plus, des pourcentages très élevés. Si l'on excepte le livre de Goudaillier où les os sont absents, les pourcentages des trois autres sources sont assez proches : 5,7\%, $7,4 \%$ et $11 \%$.

Si l'on considère les nombres des parties du corps dans chacune de nos quatre sources secondaires, les dénominations d'os par rapport aux dénominations de celles-ci représentent de $0 \%$ à $13 \%$. L'écart entre les extrêmes grandit encore. Si l'on ne tient pas compte du $0 \%$ de Goudaillier, cela va quasiment du simple (1,9\%

\footnotetext{
${ }^{7}$ Pour la raison exposée dans une note précédente (3), il n'a pas été possible de procéder à ce calcul.
} 
dans Colin) au septuple (13\% dans Guiraud) avec Mondouïs en intermédiaire à $4 \%$. Le nombre important des noms de parties du corps dans le dictionnaire de Colin avec de nombreux synonymes (1055 en tout sauf erreur) explique ce faible pourcentage, d'autant plus que, comme indiqué précédemment, les synonymes des noms d'os sont rares. Mais même le pourcentage le plus élevé reste relativement faible.

\subsection{Pourquoi si peu de noms d'os en argot / français familier ?}

Cette (quasi-)absence des noms d'os en français non conventionnel ne cesse d'intriguer. Comment l'expliquer ? On pourrait risquer l'hypothèse que les argotiers ont des mots pour ce qui est directement perceptible, les intéresse et correspond à leur expérience de la vie quotidienne. Pour le corps, c'est l'enveloppe extérieure qui est quasi-exclusivement présente, celle qui reçoit les coups, celle où sont situés les organes sensoriels (le nez pour l'odorat, sens plus développé que les autres) et les parties érotiques (seins et parties génitales). Ce sont toutes des parties tournées vers l'extérieur, directement visibles et perceptibles.

Ce qui est interne et moins directement perceptible en revanche est peu présent ${ }^{8}$ : les muscles (sauf biscottos pour biceps mais on peut les bander ostensiblement pour montrer sa force), voire complètement absent pour divers organes comme le foie ${ }^{9}$, l'œsophage, le pancréas, les intestins, la vésicule, la vessie, etc. Ne font guère exception à cette absence de dénomination argotique ou populaire des organes internes que ceux facilement et régulièrement perceptibles de l'extérieur, comme le cœur avec ses battements (les dénominations battant, palpitant sont explicites de ce point de vue) ou les poumons avec leur gonflement et dégonflement lors de l'inspiration et de l'expiration et la perception des mouvements de l'air inspiré et expiré comme l'indique clairement la dénomination soufflant.

\subsection{Hypothèses sur les quelques exceptions : les os présents. Pourquoi ceux- ci ?}

Un des rares cas où apparaissent des os sont les côtes qui suivent le mouvement d'inspiration et expiration des poumons. Ces os sont donc assez perceptibles de l'extérieur, même en étant internes. Le fait que la cage thoracique enferme et protège des organes vitaux, nobles, comme le cœur et les poumons, dont le fonctionnement est manifeste et perceptible, même en dehors des cas de douleurs et de maladies, explique sans doute aussi qu'elle fasse exception et reçoive plusieurs dénominations argotiques. À côté de la réification en termes de boucherie et d'alimentation (côtelette), ce sont des métaphores qui sont à l'œuvre. L'ensemble des côtes formant la cage thoracique sont assimilées à des cerceaux ou des cercles de barrique, du fait

\footnotetext{
${ }^{8}$ Remarquons qu'il y a néanmoins plusieurs synonymes argotiques pour le sang qui circule dans le corps, mais cela est probablement dû au fait qu'il devienne visible lors de coupures, de saignements de nez (après un «bourre-pif » par exemple), etc.

9 Mais, en loucherbem, loific est utilisé lorsque cet organe manifeste sa présence par des dysfonctionnements, souvent dus à des excès de nourriture et de boisson.
} 
de leur forme globalement arrondie. Elles sont aussi assimilées à des arêtes, du fait de leur disposition parallèle.

Le cas le plus fréquemment attesté et qui présente le plus de dénominations est celui de la tête (ou du crâne), avec toute la série synonymique des pots. Là encore c'est une métaphore qui est reconnaissable. Le mot tête lui-même n'est-il pas issu du mot latin testa dénommant un fragment de poterie / une poterie ? Plusieurs similitudes expliquent cette dénomination et celles que l'argot a développées ensuite. La forme sphérique est commune, comme l'est aussi la rigidité, qui en fait des objets cassables. Enfin ce sont des contenants. Le principe des séries synonymiques (voir supra) explique aussi la multiplication de ces dénominations qui forment une série ouverte, même si, dans les faits, ce sont les mêmes éléments qui reviennent le plus souvent.

Les deux autres cas présents ne le sont qu'épisodiquement et grime « ensemble du squelette » présent dans une seule des sources, Colin, est donné comme vieilli, hors d'usage. Seul ce dictionnaire indique deux dénominations populaires ou argotiques des mâchoires : mandibules et ganache, pour la mâchoire inférieure. Ce mot, d'abord appliqué à la mâchoire inférieure du cheval et étendu aux humains, ne semble pas très fréquent. Wikipédia (consultée le 23/06/2021) indique qu'il signifie aussi «visage" dans des emplois régionaux et aussi dans «le langage figuré moderne [...] utilisé de plus en plus en plus dans le rap français ». Remarquons encore que mandibule relève du vocabulaire courant, non marqué, dans Le Petit Robert à l'exception de l'expression donnée comme familière jouer des mandibules « manger».

\section{Des noms savants d'os en argot et ailleurs}

La pénurie de noms argotiques d'os est peut-être à l'origine de l'utilisation d'un nom scientifique d'origine savante dans le titre d'un livre écrit, en partie en argot, par A. Sarrazin : L'Astragale, publié en 1965 aux éditions Jean-Jacques Pauvert. C'est la fracture de cet os du talon - qui ne serait probablement pas très connu sans ce livre - lors du franchissement par l'autrice d'un mur pour s'échapper de prison qui constitue le point de départ du récit qui met en scène des personnages (prostituées, repris de justice, etc.) plus susceptibles d'employer l'argot qu'une langue châtiée et des mots savants.

Une autre curiosité de cohabitation de mots savants et de français non conventionnel se trouve dans une expression. Ainsi le dictionnaire de Rey et Chantreau (1994) donne, à côté des expressions du français courant se caler les amygdales «bien manger» et s'humecter les amygdales «boire (du vin, de l'alcool) » associant un mot savant (grec) et un mot courant, une expression familière (plus qu'argotique) se lécher (ou se sucer) les amygdales « échanger des baisers profonds ». «La pénétration de la langue, écrivent les auteurs, est exprimée comiquement et hyperboliquement par un organe profond de la bouche. »

À propos de noms savants d'os venant du latin et /ou du grec, il est intéressant de noter aussi, même si cela nous éloigne un peu de notre sujet, que de nouveaux noms d'os ont été introduits récemment dans Le Petit Robert. Une recherche, 
indépendante de cette contribution et antérieure, sur la relatinisation du français contemporain a en effet fait découvrir l'introduction dans ce dictionnaire de six mots latins pour des réalités déjà nommées, en particulier dans le domaine médical dont quatre en anatomie et trois pour des os : fibula, patella et scapula pour péroné, rotule et omoplate, datés respectivement de 1693, 1634 et 1734 et entrés dans les millésimes 2015 ou 2016.

\section{Conclusion}

Toutes les sources secondaires consultées concordent à confirmer l'intuition originelle de la rareté des noms d'os en argot et français populaire, sans doute parce qu'étant internes et non directement perceptibles, par la vue en particulier, ils ne se font pas remarquer, à l'opposé de ce qui est à la surface. Les deux exceptions principales, les côtes et la tête, présentes dans trois sources sur quatre, s'expliquent sans doute du fait de la place éminente de la tête et des mouvements alternés des côtes lors de la respiration. Il est remarquable que le parler actuel des jeunes de banlieue ne retienne aucun nom d'os. L'étude de données primaires, romans, chansons, films, etc. mériterait d'être conduite pour vérifier si cette quasi-absence des noms d'os des sources secondaires correspond bien à l'usage des argotiers, et si se manifestent des variations à ce sujet, en fonction de divers paramètres : âges, lieux, etc.

\section{BIBLIOGRAPHIE}

Colin Jean-Paul, MÉVEL Jean-Pierre, LeClère Christian, ([1990], 2006), Grand dictionnaire de l'argot, Paris, Larousse.

GOUDAILLIER Jean-Pierre (1997), Comment tu tchatches ! Dictionnaire du français contemporain des cités (préface de Claude HAGĖGE), Paris, Maisonneuve et Larose.

GUIRAUD Pierre ([1956] 1973), L'argot, (6 ${ }^{\mathrm{ème}}$ éd.), Que sais-Je ? nº 700, Paris, PUF.

MeILlet Antoine ([1906] 2015), "Comment les mots changent de sens », Linguistique historique et linguistique générale, Limoges, Lambert-Lucas, p. [230-271] p. 308-349.

MoNDOUÏs, Jean-Claude, Dictionnaire d'argot en ligne: https://mondouis.pagesperso-orange.fr/

Rey Alain, Chantreau Sophie (1994), Dictionnaire des expressions et locutions, ( $2^{\text {ème }}$ éd.), Paris, Le Robert.

SCHWOB Marcel, GUIEYSSE Georges (1889), Étude sur l'argot français, Mémoires de la société de linguistique de Paris, VII. 